\title{
IONIZATION MODELS OF PLANETARY NEBULAE
}

J. Patrick Harrington

Astronomy Program, University of Maryland, College Park, USA

\section{INTRODUCTION}

Our knowledge of the ionization structure of gaseous nebulae may be dated from the classic paper of Strömgren (1939), who showed that the transition between ionized and neutral hydrogen is sharp. In fact the very sharpness of the transition region has at last become a difficulty. Another milestone was the paper of Hummer and Seaton (1964) which clarified the structure of nebulae containing helium as well as hydrogen. Then, just a decade ago, the first models of planetary nebulae were constructed which detailed the changes in the temperature and in the ionization of the heavy elements as the stellar radiation was progressively diminished by absorption in the nebular gas (Goodson, 1967

Harrington, 1968; Williams, 1968; Flower, 1969a). These first models were of a general nature, but soon models were constructed to match specific nebulae (Flower, 1968, 1969b; Harrington, 1969; Kirkpatrick, 1970).

To make a model we assume knowledge of the stellar flux and of the chemical composition and three-dimensional density distribution of the gas and then solve for the temperature, the ionization and finally the intensities of the emission lines. If we are trying to fit a particular object, we will iterate on the initial assumptions to obtain an improved model. The energy and ionization equations to be solved are familiar and non-controversial. The major difference between authors is usually the degree of approximation in treating the diffuse radiation generated within the gas itself, i.e., whether one uses the "on-the-spot" approximation, the "outward only" approximation, or actually solves the transfer equation and iterates to a self-consistent solution.

Up to 1973, such models were published by Flower $(1968,1969 b)$, Harrington (1969), Kirkpatrick (1970, 1972), and Buerger(1973). Five different nebulae were modeled: NGC 7662, IC 418, NGC 7009, IC 4593, and IC 3568. The situation at the time of the Liège Symposium was reviewed by Hummer and Seaton (1973). In the last four years, however, few new objects have been attempted. 


\section{RECENT WORK}

Webster (1976) has calculated an extensive grid of model nebulae to determine the relation between gross observable features such as excitation class and the physical parameters: stellar temperature and gravity, the geometrical dilution of the radiation and the chemical composition of the nebular gas. The Hummer and Mihalas (1970) model atmospheres with temperatures from $30,000 \mathrm{~K}$ to $150,000 \mathrm{~K}$ were used to represent the central star. A good relationship between the excitation class of the nebula and stellar temperature was found for cooler stars, but the situation becomes more ambiguous for hotter stars. It also appears that the standard methods for obtaining helium and oxygen abundances can be reliable under a variety of conditions, but estimates of the nitrogen abundance based on the [N II] $6584 / \mathrm{H} \alpha$ ratio can be very unreliable.

Shields (1975) has undertaken the difficult task of modeling NGC 7027 for the purpose of obtaining the iron abundance. The atomic data for iron is unfortunately quite uncertain. From the intensity of [Fe VII] 6087 he concludes that iron is underabundant by over an order of magnitude relative to the solar value, and he attributes this to condensation into grains.

The rocket ultraviolet observations of the C IV 1549 and the C III] 1909 lines in NGC 7027 reported by Bohlin, Marionni, and Stecher (1975) provide both a challenge and an important opportunity. In high excitation nebulae carbon is as important as oxygen in cooling the gas and models where the carbon abundance is not constrained by observed lines must remain rather arbitrary. A model which is able to fit the ultraviolet carbon lines is more convincing and also enables us to determine the carbon abundance. Bohlin, Marionni, and Stecher constructed a homogeneous model which did fit the C IV 1549 line as well as the higher excitation lines in the visible spectrum such as [0 III], but the predicted C III] 1909 and the visible lines of [O II] and [N II] were too weak. This of course might be expected for an object with such complex structure. Their model does indicate that the carbon to oxygen ratio is not less than solar and is possibly higher.

Recently, Shields (1977) has again modeled NGC 7027 and extracted abundances for carbon as well as other elements. The models consist of optically thin filaments; one has about solar carbon abundance while a second model has carbon ten times solar. The failure of the models to predict sufficiently strong [O II] lines is discussed and he concludes that time dependent effects due to gas flowing into shadows is a promising explanation. The carbon abundance Shields obtains is high: $\mathrm{C} / \mathrm{H}=$ $2.5 \cdot 10^{-3}$. Unfortunately, both models, but especially the one with the high carbon abundance, seem too cool and fail to predict the proper

[O III] 5007/4363 line ratio. Although collisional de-excitation of [0 III] 5007 complicates the comparison, the fit of the models to even the high excitation lines cannot be regarded as very successful.

Bohlin, Harrington, and Stecher (1977) have obtained rocket ultraviolet measurements of the C IV 1549, C III] 1909, He II 1640, and [Ne IV] 2440 line intensities in NGC 7662 and have constructed a new 
model of this nebula which fits the ultraviolet and the visual observations quite well, including the lower excitation lines of [0 II] and [N II]. Key features of this model are a fragmented shell geometry, a modified stellar flux, and charge exchange between $\mathrm{C}^{3+}$ and $\mathrm{H}^{\circ}$. A carbon to oxygen ratio of unity was found.

Finally, a very different physical situation was investigated by Harrington and Marionni (1976), who modeled the planetary nebula which surrounds the variable FG Sge. Because the temperature of this star has declined drastically since the turn of the century (presumably as a result of a helium shell flash), the nebula has been deprived of any ionizing radiation and must be undergoing a phase of recombination and cooling. Thus we solved time-dependent ionization and energy equations. The nebula can be understood as evolving from an ordinary, low density planetary of moderate excitation. A high nitrogen abundance was indicated for this object.

3. PROBLEMS AND PROSPECTS

At this point we may ask why the list of modeled nebulae has grown so slowly. For there have been a number of favorable developments. The quality of both the observational data and the atomic parameters is improving. Models of low excitation planetaries (e.g., those of Buerger, 1973) do not seem to encounter great difficulties. The demonstration by Williams (1973) that the [0 I] line intensities could be explained when

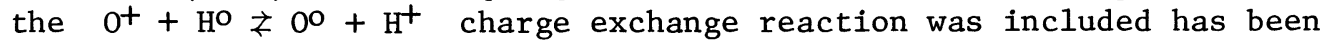
a notable success.

On the other hand ( and apart from the indolence of some of us who have the computer programs ) obstacles remain which would hinder any systematic analysis of large numbers of planetaries. First, the geometry of the model is important - uniform spherical models usually are not satisfactory. Equally important is the problem of specifying the proper stellar flux, particularly for high excitation objects. As a result, we are simultaneously varying the density distribution, the stellar flux and the chemical composition - for a change in one variable affects the others - and we inevitably end by making a large number of models and using a great deal of computer time.

The central problem, however - which is becoming an embarrassment in its obstinate refusal to be exorcised - is how to produce sufficiently intense lines from singly ionized atoms in high excitation nebulae. This applies to [N II] and [S II] but is most apparent for [O II] because the measured [0 III] line intensities prevent us from simply raising the oxygen abundance and collisional de-excitation prevents us from using condensations of arbitrarily high density. An early hope was that the shadows behind neutral globules would solve the problem, but Hummer and Seaton (1973) have shown that the intensity contributed by such shadows is limited by the number and energy of the diffuse photons. Detailed computations generally result in shadow temperatures too low to explain the observed line strengths. For the case of H II regions, Mathis (1976) has modeled such shadows, treating the transfer problem carefully, and finds that they are not satisfactory. 
Another refuge for the unsatisfactory model has been the appeal to dynamical effects as the gas flows through an ionization front. I have recently looked at the departures from ionization and thermal equilibrium in D-type ionization fronts and found that the temperature overshoot is small (Harrington, 1977). So this does not seem to be a likely solution. That is not to say that the globules are not there - we see them on photographs (Capriotti, Cromwe11, and Williams, 1971) - but we probably can represent the spectrum from their front surfaces by an isobaric, steady-state ionization front.

Aside from the shadows, the only place in a high excitation nebula where we can have much singly ionized oxygen is in the $\mathrm{H}^{+}-\mathrm{H}^{\mathrm{O}}$ transition region. If we call the thickness of this region $t$, then we have $t=30 /\left(\bar{\kappa} n_{H}\right)$, where $n_{H}$ is the total hydrogen density and $\bar{\kappa}$ is the mean opacity of hydrogen at the frequencies of the photons reaching the ionization front (the dimensionless constant is appropriate for the thickness of the region over which the ionization goes from $95 \%$ to $5 \%$ ). Consider a simple model: the nebula is a section of a shell of uniform density with thickness $\mathrm{T}$ which subtends a solid angle $\Omega$ when viewed from the central star (Figure 1 ).

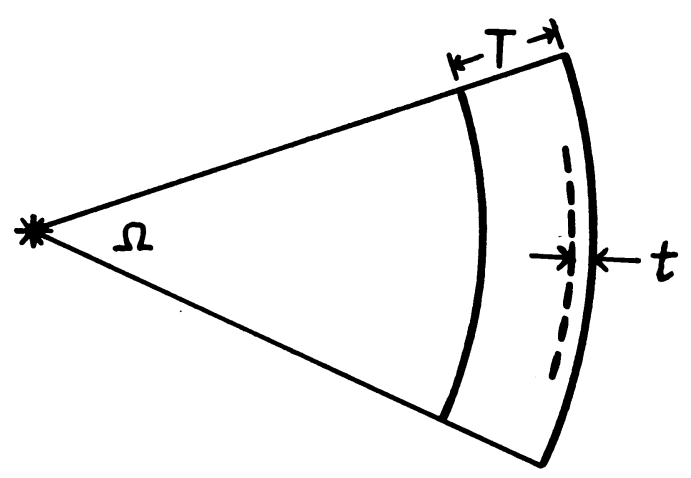

Figure 1. Geometry of the model.

Since observations will constrain a model to have a definite surface brightness, we can only vary the volume and density in such a way that this quantity remains constant: $\Omega \mathrm{T} \mathrm{n}_{\mathrm{H}}^{2}=$ constant. Then, if this shel1 contains a transition region, the fraction of the nebula occupied by this region is

$$
(\mathrm{t} / \mathrm{T}) \propto \Omega \mathrm{n}_{\mathrm{H}} / \overline{\mathrm{K}} .
$$

To increase the [0 II] line intensity, we want to make $(t / T)$ as large as possible. We can increase the density, $n_{H}$, but this will not be effective after collisional de-excitation becomes important. In fact, in a well observed nebula, the density sensitive [0 II] $\lambda 3729 / \lambda 3726$ line ratio will determine the appropriate value of $\mathrm{n}_{\mathrm{H}}$. Putting high density condensations into the model is essentially equivalent to increasing $\mathrm{n}_{\mathrm{H}}$ and encounters the same limitations.

We will try to make $\Omega$ as large as possible, and since our shell has an ionization front, we are thus making our nebula optically thick at 
the Lyman limit. Here we may encounter a problem with the stellar flux. Many nebulae have a He II Zanstra temperature which is much higher than the $\mathrm{H}$ I Zanstra temperature, and this is usually regarded as evidence that the nebula is not optically thick to hydrogen ionizing radiation. The available model atmosphere fluxes make matters worse because of the large drop in flux at $\lambda 228 \AA$ which they display. If we want to get the He II line intensities correct and still surround our star with an ionization front, we must have a flux distribution with a deficiency of photons between $\lambda 912$ and $\lambda 228$.

Finally, note that it may help to have more high frequency radiation because this makes $\bar{\kappa}$ smaller. We can, in fact, produce a large increase in $(t / T)$ if we make the spectrum so flat that the photons between $\lambda 912$ and $\lambda 228$ are exhausted before those at $\lambda<228 \mathrm{~A}$ and the hydrogen competes with ionized helium for this high frequency radiation. Then $\bar{K}$ drops and we have an exotic transition region with $\mathrm{He}^{++}$and $\mathrm{H}^{\mathrm{O}}$ coexisting.

The point I wish to make is that if we do not invoke time-dependent effects or introduce new physical processes, modifying the stellar flux is the only way I see to enhance the low excitation lines - the modification, paradoxically, is to make the spectrum harder. I think this should at least be explored. At this stage we should not stand too much in awe of the model atmosphere calculations. I might note here Kaler's (1976) paper which shows that blackbodies define a better temperature for the central star by Stoy's method than the atmospheres do.

In our model of NGC 7662 (Bohlin, Harrington, and Stecher, 1977), we were able to fit the [O II] lines by a combination of shell geometry and a modified stellar flux. The resultant increase in the volume of the transition region is accompanied by about $1 \%$ neutral hydrogen at the center of the outer she11, and this is why charge exchange becomes important. When we included the $\mathrm{N}^{3+}+\mathrm{H}^{\mathrm{O}} \rightarrow \mathrm{N}^{2+}+\mathrm{H}^{+}$reaction in the model, using the rate of Christensen, Watson, and Blint (1977), there was an increase in the intensities of the [N II] lines of $60 \%$. It is not obvious that a process which depends upon neutral hydrogen will be important in high excitation planetary nebulae, but when you have constructed a model to produce the observed low excitation lines, you have also created the conditions where such effects are going to be important. As yet we have a very incomplete knowledge of charge exchange rates, but it is already clear that they may be very important for ionization models.

\section{REFERENCES}

Bohlin,R.C., Harrington,J.P., and Stecher, T.P.: 1977, "The RocketU1traviolet Spectrum and Models of the Planetary Nebula NGC 7662", Astrophys.J. (in press).

Bohlin,R.C., Marionni,P.A., and Stecher,T.P.: 1975, Astrophys.J. 202,415. Buerger, E.: 1973, Astrophys. J. 180, 817.

Capriotti,E.R., Cromwe11,R.H., and Williams,R.E.: 1971, Astrophys.Letters $\underline{7}, 241$.

Christensen,R.B., Watson,W.D., and Blint,R.J.: 1977, Astrophys.J. 213, 712 . Flower,D.R.: 1968, Astrophys. Letters 2, 205.

Flower,D.R.: 1969a, Monthly Notices Roy. Astron. Soc. 146,171.

Flower,D.R.: 1969b, Monthly Notices Roy. Astron. Soc. $146,243$.

Goodson,W.L. : 1967 , Z. Astrophys . 66,118 . 
Harrington, J.P.: 1968, Astrophys.J. 152,943.

Harrington, J.P.: 1969, Astrophys.J. $\overline{156}, 903$.

Harrington, J.P.: 1977, Monthly Notices Roy. Astron. Soc. 179,63.

Harrington, J.P., and Marionni,P.A.: 1976, Astrophys.J. 206,458.

Hummer,D.G. and Mihalas, D.: 1970, Monthly Notices Roy. Astron. Soc. 147,339 .

Hummer,D.G. and Seaton,M.J.: 1964 , Monthly Notices Roy. Astron. Soc. $\underline{127}, 217$.

Hummer,D.G. and Seaton,M.J.: 1973, Mem. Soc. Roy. Sci. Liege, 6e Serie $\underline{5,225}$.

Kaler,J.B.: 1976, Astrophys.J. 210, 843.

Kirkpatrick,R.C.: 1970, Astrophys.J. 162, 33.

Kirkpatrick,R.C.: 1972, Astrophys.J. $\underline{176}, 381$.

Mathis,J.E.: 1976, Astrophys.J. 207,4 $4 \overline{42}$.

Shields, G.A.: 1975, Astrophys.J. 195, 475 .

Shields,G.A.: 1977,"Ionization St Nebula NGC 7027", preprint.

Stromgren,B.: 1939, Astrophys.J. 89, 526.

Webster,B.L.: 1976, Monthly Notices Roy.Astron. Soc. 174,157.

Williams,R.E.: 1968, I.A.U. Symposium No. 34 "Planetary Nebulae", ed.

D.E. Osterbrock and C.R. O'Dell (D.Reidel; Dordrecht, Holland), 190. Williams,R.E. 1973, Monthly Notices Roy. Astron. Soc. 164,111.

\section{DISCUSSION}

Dopita: Have you considered the possibility that the strong [OII] lines can be produced by shock waves beyond the ionization front? These could be generated either by shock detachment during the transition from $r$-type to d-type front, or more likely by an accelerating mass flow from the central star before appreciable ionizing radiation is produced by this object.

Harrington: No, I haven't looked into the shocks. I think you have an energy problem because the planetary nebula is extremely efficient in that it converts a large fraction of the stellar luminosity into forbidden line radiation.

Zuckerman: Does the [OII] problem occur often enough to rule out a time-dependent effect such as the decrease in the luminosity of the underlying star?

Harrington: I think it's pretty prevalent. I have abandoned trying to make models of a number of objects just by glancing at the [OII] line strengths and seeing I didn't have a chance of fitting them. I would be surprised if you could explain this sort of difficulty with time-dependent effects.

Aller: In the end, we may be forced to modify the central star energy distribution, but I'd like to see us apply all the pertinent physics we can first. Extensive model calculations carried out at UCLA by C.D. Keyes demonstrated to our.satisfaction that homogeneous models fail 
miserably for all planetaries so far tried. Even inhomogeneities in density, etc., don't suffice to remove discordances. For example, in our models, HeII $\lambda 4686$ tends to be predicted too weak. Modifications of the stellar energy distribution as suggested by Harrington would seem to be required. I cannot agree more that we are terribly ignorant about the UV stellar energy distributions, so all the nebular diagnostic data we can get is going to be helpful in narrowing the range. 